\title{
BRAF MUTATIONS IN HAIRY CELL LEUKEMIA
}

\author{
Enrico Tiacci, M.D., Vladimir Trifonov, Ph.D., Gianluca Schiavoni, Ph.D., Antony Holmes, \\ Ph.D., Wolfgang Kern, M.D., Maria Paola Martelli, M.D., Alessandra Pucciarini, Ph.D., \\ Barbara Bigerna, B.Sc, Roberta Pacini, B.Sc., Victoria Wells, B.Sc., Paolo Sportoletti, M.D., \\ Valentina Pettirossi, Ph.D., Roberta Mannucci, Ph.D., Oliver Elliott, Ph.D., Arcangelo Liso, \\ M.D., Achille Ambrosetti, M.D., Alessandro Pulsoni, M.D., Francesco Forconi, M.D., Livio \\ Trentin, M.D., Gianpietro Semenzato, M.D., Giorgio Inghirami, M.D., Monia Capponi, M.D., \\ Francesco Di Raimondo, M.D., Caterina Patti, M.D., Luca Arcaini, M.D., Pellegrino Musto, \\ M.D., Stefano Pileri, M.D., Claudia Haferlach, M.D., Susanne Schnittger, Ph.D., Giovanni \\ Pizzolo, M.D., Robin Foà, M.D., Laurent Farinelli, Ph.D., Torsten Haferlach, M.D., Laura \\ Pasqualucci, M.D., Raul Rabadan, Ph.D., and Brunangelo Falini, M.D. \\ Institute of Hematology, University of Perugia, Perugia, Italy (E.T., G.S., M.P.M., A.P., B.B., R.P., \\ P.S., V.P., M.C., L.P., B.F.); Department of Biomedical Informatics and Center for Computational \\ Biology and Bioinformatics, Columbia University (V.T., A.H., O.E., R.R.); Munich Leukemia \\ Laboratory (MLL), Munich, Germany (W.K., C.H., S.S., T.H.); Institute for Cancer Genetics (V.W., \\ L.P.), Columbia University, New York, NY, USA; Institute of Internal Medicine and Oncologic \\ Sciences, University of Perugia, Perugia, Italy (R.M.); Institute of Hematology, University of \\ Foggia, Foggia, Italy (A.L.); Department of Medicine, Hematology Section, University of Verona, \\ Verona, Italy (A.A., G.P.); Division of Hematology, Department of Cellular Biotechnologies and \\ Hematology, Sapienza University, Rome, Italy (A.P., R.F.); Hematology, University of Siena \& \\ AOUS, Siena, Italy (F.F.); Department of Clinical and Experimental Medicine, Hematology and \\ Clinical Immunology Section, University of Padua, Padua, Italy (L.T., G.S.); Department of \\ Pathology, Center for Experimental Research and Medical Studies, University of Torino, Turin, \\ Italy (G.I.); Section of Hematology, Department of Biomedical Sciences, Ferrarotto Hospital, \\ University of Catania, Catania, Italy (F.D.R); Department of Hematology, Cervello Hospital, \\ Palermo, Italy (C.P.); Division of Hematology, Department of Oncohematology, University of \\ Pavia Medical School, Fondazione IRCCS Policlinico S. Matteo, Pavia, Italy (L.A.); IRCCS, \\ Centro di Riferimento Oncologico della Basilicata (CROB), Rionero in Vulture/Potenza, Italy \\ (P.M.); Section of Hemopathology, Department of Hematology and Oncologcal Sciences \\ "Seragnoli", University of Bologna, Bologna, Italy (S.P.); Fasteris SA, Plan-les-Ouates/Geneva, \\ Switzerland (L.F.)
}

\section{Abstract}

Address correspondence to: Dr. B. Falini (Institute of Hematology, University of Perugia, Ospedale S. Maria della Misericordia, S. Andrea delle Fratte, 06132 Perugia, Italy; faliniem@ unipg.it) or Dr. R. Rabadan (Department of Biomedical Informatics and Center for Computational Biology and Bioinformatics, Columbia University, 1130 St. Nicholas Ave, ICRC Bldg 8th Floor, Office 803B, New York, NY 10032, USA; rabadan@dbmi.columbia.edu).

Authors' contribution

BF designed the study, supervised the project, and wrote the paper. E.T. designed the study, designed and performed wet laboratory experiments, and wrote the paper. V.T. and R.R. designed and performed the bioinformatic analyses and contributed to write the paper. L.P. supervised bioinformatic analysis validation and contributed to write the paper. L.F. supervised whole exome sequencing. G.Sc., M.P.M, A.Puc., B.B., R.P., V.W., P.S., V.P. and R.M. designed and performed wet laboratory experiments. A.H. and O.E. designed and performed bioinformatic analyses. All other authors (W.K., A.L., A.A., A.Pul., F.F., L.T., G.Se.., G.I., M.C., F.D.R., C.P., L.A., P.M., S.P., C.H., S.S., G.P., R.F. and T.H) provided patients' samples as well as immunophenotypic and clinical data. All authors vouch for the respective data and analyses, and agreed to publish the paper. A patent application on the clinical use of BRAF mutants in $\mathrm{HCL}$ is pending. 
Background-Hairy cell leukemia (HCL) is a well defined clinico-pathological entity whose underlying genetic lesion is still obscure.

Methods-We searched for HCL-associated mutations by massively parallel sequencing of the whole exome of leukemic and matched normal mononuclear cells purified from the peripheral blood of one patient with HCL.

Results-Whole exome sequencing identified 5 missense somatic clonal mutations that were confirmed at Sanger sequencing, including a heterozygous V600E mutation involving the $B R A F$ gene. Since the $B R A F \mathrm{~V} 600 \mathrm{E}$ mutation is oncogenic in other tumors, further analyses were focused on this genetic lesion. Sanger sequencing detected mutated $B R A F$ in $46 / 46$ additional HCL patients (47/47 including the index case; $100 \%)$. None of the 193 peripheral B-cell lymphomas/leukemias other than HCL that were investigated carried the BRAFV600E mutation, including 36 cases of splenic marginal zone lymphomas and unclassifiable splenic lymphomas/ leukemias. Immunohistological and Western blot studies showed that HCL cells express phosphoMEK and phospho-ERK (the downstream targets of the BRAF kinase), indicating a constitutive activation of the RAF-MEK-ERK mitogen-activated protein kinase pathway in HCL. In vitro incubation of $B R A F$-mutated primary leukemic cells from 5 HCL patients with PLX-4720, a specific inhibitor of active BRAF, led to marked decrease of phosphorylated ERK and MEK.

Conclusions-The BRAFV600E mutation was present in all HCL patients investigated. This finding may have relevant implications for the pathogenesis, diagnosis and targeted therapy of HCL (Funded by the Associazione Italiana Ricerca Cancro and others).

\section{INTRODUCTION}

Hairy cell leukemia (HCL) represents a distinct disease entity characterized by indolent course, marked splenomegaly, frequent progressive pancytopenia, rare circulating tumor cells, and usually no lymphadenopathy(1). Bone marrow, spleen and liver are characteristically infiltrated by leukemic B cells showing abundant cytoplasm with "hairy" projections and unique immunophenotypic features $(2,3)$. Currently, treatment of HCL is based upon highly effective purine nucleoside analogs(4).

In spite of the remarkable progress in the diagnosis and treatment of HCL over the past 50 years, its underlying genetic alterations remain obscure (3). Major obstacles to in depth molecular characterization of HCL have been the scarcity of tumor cells available for analysis (due to frequent pancytopenia), the very low proliferative index of leukemic cells, the inability to grow them in immunodeficient mice and the absence of human cell lines of well proven HCL origin.

No recurrent chromosomal translocations have been identified in HCL(1). Gene expression profiling studies revealed a unique molecular signature that in part justifies the distinctive features of HCL cells, like their morphological appearance, adhesion properties, selective homing to extranodal sites and marrow fibrosis(5). However, these studies did not pinpoint any recurrent genetic alteration. Similarly, high density genome-wide SNP genotyping showed a remarkably balanced genomic profile in $\mathrm{HCL}(6)$.

A powerful approach to better understand the genetic basis of cancer is offered by genomewide massively parallel sequencing of tumor and normal cells from the same patient aimed to identify novel acquired alterations $(7,8)$. We performed in-solution exome capture followed by massively parallel sequencing to investigate the DNA of purified peripheral blood (PB) leukemic and paired normal mononuclear cells from a HCL patient. Our goal was to identify potential recurrent somatic mutations in protein-coding genes which could shed light on the biology of HCL and offer new targets for diagnosis and treatment. 


\section{MATERIALS AND METHODS}

\section{Tumor and normal samples}

Criteria for HCL diagnosis were according to the WHO 2008 classification(1). All 47 cases showed the typical clinical picture and morphology of HCL, were positive for Annexin A1(2) at immunohistochemistry and/or co-expressed CD11c, CD25 and CD103 at flow cytometry.

Whole exome sequencing (WES) in the index HCL patient was performed on purified (>90\%) CD19-positive leukemic cells at disease onset and purified (>98\%) CD19-negative PB mononuclear cells after chemotherapy (Supplementary Materials). The patient gave written informed consent for WES analysis under a protocol approved by the Ethics Committee, University of Perugia. All patients gave informed consent for the analysis of their sample material.

\section{WES and bioinformatic analyses}

Preparation of shotgun libraries from the leukemic and non-leukemic genomic DNA of the index patient, followed by in-solution exome capture, was performed using a commercial platform (Agilent) covering $38 \mathrm{Mb}$ of coding exons ( 1.22\% of the genome). After massively parallel sequencing with the Illumina Genome Analyzer IIx, candidate somatic mutations were identified according to a bioinformatic pipeline (Fig. 1 and Supplementary Materials).

\section{Validation and screening of candidate mutations by PCR amplification and Sanger sequencing}

Candidate non-synonymous somatic variants (present in the tumor but not in its paired normal DNA) were verified by PCR amplification and direct DNA sequencing of the same patient samples subjected to WES (Tables S1 and S2). Because the sensitivity of Sanger sequencing allows to detect heterozygous mutations only when present in a major clonal population, sequence variants reported in $<25 \%$ of the reads were not included in this validation phase.

PCR and direct DNA Sanger sequencing of $B R A F$ exon-15 in an extended cohort of HCL patients $(n=46)$ or other peripheral B-cell lymphomas/leukemias $(n=193)$ were performed using the primers e $15 \mathrm{~F}-5^{\prime}$-TACCTAAACTCTTCATAATGCTTGC- $3^{\prime}$ and e15R-5' GTAACTCAGCAGCATCTCAGGG-3'. Cycling conditions (after the initial denaturation step at $94^{\circ} \mathrm{C}$ for $2^{\prime}$ ) were $94^{\circ} \mathrm{C}$ for $30^{\prime \prime}, 56^{\circ} \mathrm{C}$ for $30^{\prime \prime}, 72^{\circ} \mathrm{C}$ for $20^{\prime \prime}$ for 40 cycles, followed by a final elongation at $72^{\circ} \mathrm{C}$ for $10^{\prime}$. For 48 diffuse large B-cell lymphomas (DLBCLs) primers and cycling conditions are reported in Tables S1 and S2. All 239 patient samples investigated had $\geq 30 \%$ neoplastic cells.

\section{Immunohistological and Western blotting studies}

Details of the immunohistological and Western blot (WB) studies for phospho-ERK and phospho-MEK in HCL samples (under basal conditions and following in vitro incubation with active BRAF inhibitors) are given in the Supplementary Materials.

\section{RESULTS \\ Case report}

A 47 year-old male presented with fever and pneumonia in March 2009. Laboratory data showed $\mathrm{Hb} 11.5 \mathrm{~g} / \mathrm{dL}$, WBC $6,100 / \mathrm{mm}^{3}$ (49\% circulating leukemic hairy cells) and platelets $70,000 / \mathrm{mm}^{3}$. Splenomegaly was present. A bone marrow trephine showed a typical HCL 
histology. At immunohistochemistry, leukemic cells were positive for CD20, DBA44, CD68 and Annexin-A1; expression of CD20, CD11c, CD25 and CD103 was documented by flow cytometry. The patient was started on therapy with pentostatin (deoxycoformicin) and he is now in complete hematological remission.

\section{Identification of candidate somatic mutations in a HCL patient}

WES of genomic DNA from patient's purified leukemic and non-leukemic cells produced $\sim 42.5$ and $\sim 42.8$ million reads, respectively, of 108 nucleotides length (Table S3). After removal of low quality and duplicate reads, the mean depth of the covered exome was 71 (tumor) and 70 (normal) (median 52 and 51, respectively), with 99\% of the target exome being covered by at least one read, and $86 \%$ by at least 10 reads (Table S3). The SAVI algorithm (Supplementary Materials) identified 5 unique non-synonymous variants that were present specifically in the tumor DNA ( $27 \%$ to $49 \%$ of the reads), and targeted the $B R A F$, CSMD3, SLC5A1, CNTN6, and OR8J1 genes (Tables S4 and S5). All 5 variants were validated as somatic in origin after targeted Sanger re-sequencing of paired tumor and normal DNA from the same patient (Tables S4 and S5). All mutations were heterozygous; they were presumably clonally represented in the tumor population (as also assessed by the comparable size of the corresponding peak in the chromatogram), and introduced amino acid substitutions in the encoded proteins (Table 1). The extremely low false discovery rate (near $0 \%$ ) of the SAVI algorithm did not come at the expense of a low sensitivity. Indeed, sensitivity (proportion of true variants identified), as estimated from the number of known germline polymorphisms present in dbSNP130 and detected by the algorithm, was similar to other estimates of previous studies $(9,10)$ (Supplementary Materials and Table S6)

\section{BRAF mutations in HCL samples}

While little is known about the biological role of $4 / 5$ affected genes (Table S6 S7) BRAF represents the most frequently mutated gene encoding a protein kinase in human cancers(11). Interestingly, the same amino acid change (V600E) identified in our patient represents a mutational hotspot in melanomas and papillary thyroid cancers, where its oncogenic activity has been extensively documented $(12,13)$. Moreover, dysregulation of the mitogen-activated protein kinase (MAPK) kinase pathways, of which BRAF is a component, was previously implicated in HCL pathogenesis(14).

We, therefore, screened 46 additional HCL cases for this mutation by PCR and direct DNA Sanger sequencing of $B R A F$ exon-15. The features of these cases are shown in Table 1. Strikingly, a T->A transversion, occurring at position 1860 of the $B R A F$ mRNA RefSeq NM_004333.4 and resulting in a Val->Glu amino acid substitution at position 600 of the BRAF protein (V600E), was found in 46/46 HCL cases (47/47, including the index patient $100 \%)$. The mutation was somatic in origin, as it was absent in matched non-leukemic DNA of all $10 \mathrm{HCL}$ cases investigated.

In 29/47 mutated HCL patients (including the index case), the high purity of leukemic cells $(>90 \%)$ allowed to study the zygosity status of the mutation without the interference of the wild-type alleles contributed by the contaminating non-leukemic cells. In 25/29 cases, the mutation appeared as a double peak (Fig. 2, top panels), strongly indicating a heterozygous lesion occurring in all cells of the leukemic clone. In the remaining 4 patients, only the mutant peak was observed (Fig. 2, upper-middle panels), pointing to a homozygous/ hemizygous clonal event.

In 17/47 HCL patients, the height of the mutated peak in the chromatogram relative to the proportion of leukemic cells in the analyzed samples (from 30\% to 74\%) was consistent with the mutation being clonal. In the remaining HCL case (patient 7 of Table 1), the fresh 
PB sample, that was initially analyzed and found to be $B R A F$ wild-type, turned out retrospectively to contain a proportion of leukemic cells far below the detection threshold of direct Sanger sequencing for a heterozygous clonal mutation (about $30 \%$ in our hands). Because this HCL patient is currently in remission with $<0.1 \%$ leukemic cells circulating in the $\mathrm{PB}$, the only sample with considerable leukemic infiltration that we could investigate from this case was an archival fixed-paraffin-embedded bone marrow biopsy. Although the quantity and quality of genomic DNA extracted from this sample were very poor, we could perform Sanger sequencing on the cloned faint PCR product, and 4/26 sequenced clones were mutated. In conclusion, the $B R A F$ mutation was detected in all $47 \mathrm{HCL}$ cases analyzed and was clonal in at least 46 of them.

\section{BRAF mutation in other B-cell lymphomas/leukemias}

Given the high frequency of $B R A F \mathrm{~V} 600 \mathrm{E}$ in $\mathrm{HCL}$, we next investigated whether the same variant is also present in other peripheral B-cell lymphomas/leukemias. Strikingly, none of the 193 cases investigated carried the mutation (Table S8). BRAF-negative cases also included some B-cell tumors that, both clinically and morphologically, may simulate HCL (but have a different prognosis and clinical management), such as splenic marginal zone lymphoma (also frequently referred to as splenic lymphoma with villous lymphocytes) $(n=21)$ and splenic B-cell lymphoma/leukemia, unclassifiable $(n=15)$ (Fig. 2, lower-middle and bottom panels). The latter category includes HCL-variant and splenic red pulp small Bcell lymphoma, according to the 2008 WHO classification(1).

\section{Expression of phosphorylated MEK and ERK in leukemic hairy cells}

The BRAFV600E mutation results in the constitutive activation of its kinase activity. Therefore, we assessed the phosphorylation status of MEK (the immediate downstream kinase target of BRAF) and ERK (the kinase phosphorylated by active MEK), using antibodies that specifically recognize phosphorylated MEK and ERK.

Because phospho-epitopes can be denatured by the decalcification process, we selected for immunohistological studies 5 BRAF-mutated HCL cases for which optimally fixed/ decalcified paraffin-embedded bone marrow biopsies were available. In all cases, double immunohistochemical and/or immunofluorescence stainings for a B-cell marker (PAX5 or CD20) and phospho-ERK showed phosphorylated ERK in HCL cells, which was abolished by pre-incubation of the anti-phospho-ERK antibody with its blocking phospho-peptide (Fig. 3, top panels; Supplementary Fig. 1). Phospho-MEK could not be investigated due to the unreliable staining of the anti-phospho-MEK antibody in paraffin sections. In 2/5 BRAFmutated HCL patients that were phosho-ERK positive at immunohistochemistry a sufficient number of purified HCL cells was available for WB analysis. Both of them showed phosphorylation of MEK and its substrate ERK (data not shown). These results are in keeping with previous findings in HCL by Kamiguti et al. (14).

In-vitro incubation of primary leukemic cells from 5 additional patients with the specific active BRAF inhibitor PLX-4720 led to marked decrease of phosphorylated MEK and ERK at low $(\leq 1 \mu \mathrm{M})$ drug concentrations, while vehicle-treated cells retained MEK and ERK phosphorylation (Fig. 3, bottom panels).

\section{DISCUSSION}

By whole exome sequencing of a patient with $\mathrm{HCL}$, we identified $B R A F \mathrm{~V} 600 \mathrm{E}$ mutation as the first genetic alteration recurrently associated with this disease. The $B R A F \mathrm{~V} 600 \mathrm{E}$ mutation qualifies as the disease-defining genetic event of HCL because of: i) its presence in $100 \%$ of cases in our series encompassing the whole spectrum of HCL patients, including 
those presenting with leukocytosis or without splenomegaly and those analyzed after therapy; ii) its presence in the entire tumor cell clone in virtually all patients; and iii) its restriction to HCL among peripheral B-cell lymphomas/leukemias. This strongly implicates the BRAF V600E mutation in HCL pathogenesis. Notably, among B-cell neoplasms (in which non-kinase genes are usually involved by a variety of genetic alterations, i.e. translocations, deletions, or point mutations), HCL is the only one whose disease-defining genetic lesion is represented by an activating point mutation of a kinase-encoding gene. Interestingly, the frequency of $B R A F \mathrm{~V} 600 \mathrm{E}$ in HCL far outnumbers that previously reported for other $B R A F$-mutated human neoplasms, including melanomas $(\sim 50 \%)(11,15)$, papillary thyroid carcinomas $(\sim 40 \%)(16)$, Langherans cell histiocytosis $(57 \%)(17)$ and a variety of solid tumors (at much lower frequency)(11, 18, 19).

A member of the serine/threonine kinase RAF family, the BRAF protein is part of the RASRAF-MAPK signaling pathway which plays a major role in regulating cell survival, proliferation and differentiation(24). BRAF mutations (with V600E being the most common in human tumors) constitutively activate the MEK-ERK pathway, leading to enhanced cell proliferation, survival and ultimately, neoplastic transformation(12, 13, 25). All $B R A F$ mutated HCL cases carried the V600E phospho-mimetic substitution which occurs within the BRAF activation segment and markedly enhances its kinase activity in a constitutive manner(26).

Interestingly, activation of the MAPK pathway was previously described in $\operatorname{HCL}(14,27)$. Kamiguti et al.(14) found that ERK was constitutively activated and provided a survival signal for the leukemic hairy cells $(14,27)$. ERK-mediated survival was hypothesized to act by rescuing HCL cells from the potentially pro-apoptotic effect of p38/JNK activation (triggered by the interaction of leukemic cells with the vitronectin-positive cells in the splenic red pulp) $(14,28)$. ERK activation in HCL was reported to depend upon MEK activation by a still unclear route rather than upon RAF activation(14). Our findings implicate mutated $B R A F$ as the most likely trigger for constitutive MEK and ERK activation in HCL. This is supported by our immunohistochemical and WB analyses for phospho-MEK and phospho-ERK showing that, in the presence of $B R A F$ mutations, these downstream targets are activated in HCL cells and are dephosphorylated upon treatment with a specific active BRAF inhibitor. Based on our findings, it should be feasible to develop murine HCL models by activating the RAS-RAF-MAPK signalling pathway in specific B-cell subsets. Further studies are required to clarify whether other mutations may cooperate with $B R A F$ in HCL pathogenesis.

The $B R A F \mathrm{~V} 600 \mathrm{E}$ mutation may account for some HCL immunophenotypic features, e.g. the low/moderate cyclin-D1 expression (which is independent of $C C N D 1$ rearrangements or amplifications) $(29,30)$ and absence of p27(31). In melanoma cells, V600E BRAFleads to MEK/ERK pathway activation with concomitant transcriptional constitutive expression of cyclin-D1 and p27 downregulation in an adhesion-independent manner(32-34). Moreover, MEK-ERK-induced activation of an AP1-transcription factor complex containing JUND(35) has been implicated in the expression of the HCL marker CD11c.

$B R A F \mathrm{~V} 600 \mathrm{E}$ was present in all our $47 \mathrm{HCL}$ cases. However, given the relatively limited number of patients investigated, we cannot exclude that $B R A F \mathrm{~V} 600 \mathrm{E}$ negative HCL cases may exist. It must be also stressed that all cases analyzed in this study contained $\geq 30 \%$ neoplastic cells which, in our hands, was the threshold for detecting a heterozygous clonal mutation at direct Sanger sequencing. Detection of the $B R A F$ mutation in HCL samples containing lower numbers of leukemic cells will require more sensitive techniques. 
Among a total of 240 peripheral B-cell lymphomas studied, $B R A F \mathrm{~V} 600 \mathrm{E}$ was restricted to HCL. However, it cannot be excluded that B-cell lymphomas other than HCL may occasionally carry $B R A F$ mutations in exon- 15 but that they were not found in this study because of the limited number of cases investigated for some lymphoma subsets (Table S8). Indeed, $B R A F$ mutations have been rarely reported in B-cell acute lymphoblastic leukemias (6/213 cases)(20-22) and peripheral B-cell lymphomas (4/164 cases)(23), although they were in all instances different from V600E.

Overall our findings point to analysis of $B R A F$ mutations as a potential new diagnostic tool to distinguish HCL from other B-cell lymphomas exhibiting similar clinical and morphological features, such as HCL-variant and splenic marginal zone lymphoma, none of which was $B R A F$-mutated in this study. This distinction is clinically relevant since HCL but not HCL-like disorders respond optimally to interferon or purine analogs (4). Absence of $B R A F$ mutation in HCL-variant further supports the view that this represents an entity different from HCL and justifies its inclusion in the category of splenic B-cell lymphoma/ leukemia, unclassifiable of the 2008 WHO classification(36).

Finally, the BRAF V600E mutant is a new potential therapeutic target for those HCL patients who do not respond or respond suboptimally to initial therapy with purine analogs as well as for patients experiencing repeated relapses(4). Notably, BRAF V600E inhibitors(37-39) have shown remarkable activity in patients with $B R A F$-mutated metastatic melanoma(40). These results and our in-vitro findings that a specific active BRAF inhibitor causes MEK and ERK dephosphorylation in primary HCL cells warrant the clinical testing of active BRAF inhibitors, alone or in combination with compounds acting downstream of BRAF (e.g., MEK or ERK inhibitors), in HCL patients.

\section{Supplementary Material}

Refer to Web version on PubMed Central for supplementary material.

\section{Acknowledgments}

This work was supported by grants from the Associazione Italiana Ricerca Cancro (A.I.R.C.) including the specific "Programma di Oncologia Clinica Molecolare 5 per mille", the Associazione Umbra contro le Leucemie e i Linfomi (AULL), the Fondazione CARIVERONA, and the Hairy Cell Leukemia Research Foundation. Enrico Tiacci is supported by a fellowship (2008/14) from the European Hematology Association. The work of Trifonov, Holmes and Rabadan is supported by the Northeast Biodefense Center (U54-AI057158) and the National Library of Medicine (1R01LM010140-01). Arcangelo Liso is supported by AIL-Foggia. We thank Riccardo Dalla Favera for critically reading the manuscript; Dr. Alessia Tabarrini, Dr. Francesca Strozzini, Dr. Franca Falzetti, Dr. Elisabetta Bonifacio and Dr. Debora Cecchini for their invaluable help in immunohistochemical, Western blot and flowcytometry stainings; Dr. Magne Osteras and Dr. Nicolas Gonzalez for technical help in library preparation, exome capture and massively parallel sequencing; Dr. Loich Baerlocher and Dr. Julien Prados for excellent bioinformatic support; Prof. Stefano Ascani, Prof. Fabio Menestrina, Dr. Alberto Zamò, and Dr. Monica Facco for providing pathological material of some patients; Prof. Giorgina Specchia for providing clinical informations on one HCL patient; Geraldine A. Boyd for editing the paper and Claudia Tibidò for secretarial assistance; and our patients.

We wish to dedicate this study to Prof. Massimo F. Martelli, at the time of his retirement, to underline his lifetime commitment to the fight against leukemia and his continuous support towards the growth of the Perugia Hematology school.

\section{References}

1. Foucar, K.; Falini, B.; Catovsky, D.; Stein, H. Hairy cell leukaemia. In: Swerdlow, S.; Campo, E.; Harris, NL., et al., editors. WHO Classification of Tumours of Haematopoietic and Lymphoid Tissues. 4. Lyon: International Agency for Research on Cancer (IARC); 2008. p. 188-90. 
2. Falini B, Tiacci E, Liso A, et al. Simple diagnostic assay for hairy cell leukaemia by immunocytochemical detection of annexin A1 (ANXA1). Lancet. 2004; 363:1869-70. [PubMed: 15183626]

3. Tiacci E, Liso A, Piris M, Falini B. Evolving concepts in the pathogenesis of hairy-cell leukaemia. Nat Rev Cancer. 2006; 6:437-48. [PubMed: 16723990]

4. Grever MR. How I treat hairy cell leukemia. Blood. 2010; 115:21-8. [PubMed: 19843881]

5. Basso K, Liso A, Tiacci E, et al. Gene expression profiling of hairy cell leukemia reveals a phenotype related to memory B cells with altered expression of chemokine and adhesion receptors. J Exp Med. 2004; 199:59-68. [PubMed: 14707115]

6. Forconi F, Poretti G, Kwee I, et al. High density genome-wide DNA profiling reveals a remarkably stable profile in hairy cell leukaemia. Br J Haematol. 2008; 141:622-30. [PubMed: 18397341]

7. Campbell PJ, Stephens PJ, Pleasance ED, et al. Identification of somatically acquired rearrangements in cancer using genome-wide massively parallel paired-end sequencing. Nat Genet. 2008; 40:722-9. [PubMed: 18438408]

8. Ley TJ, Mardis ER, Ding L, et al. DNA sequencing of a cytogenetically normal acute myeloid leukaemia genome. Nature. 2008; 456:66-72. [PubMed: 18987736]

9. Choi M, Scholl UI, Ji W, et al. Genetic diagnosis by whole exome capture and massively parallel DNA sequencing. Proc Natl Acad Sci U S A. 2009; 106:19096-101. [PubMed: 19861545]

10. Ng PC, Levy S, Huang J, et al. Genetic variation in an individual human exome. PLoS Genet. 2008; 4:e1000160. [PubMed: 18704161]

11. Davies H, Bignell GR, Cox C, et al. Mutations of the BRAF gene in human cancer. Nature. 2002; 417:949-54. [PubMed: 12068308]

12. Wellbrock C, Hurlstone A. BRAF as therapeutic target in melanoma. Biochem Pharmacol. 2010; 80:561-7. [PubMed: 20350535]

13. Li Y, Nakamura M, Kakudo K. Targeting of the BRAF gene in papillary thyroid carcinoma (review). Oncol Rep. 2009; 22:671-81. [PubMed: 19724843]

14. Kamiguti AS, Harris RJ, Slupsky JR, et al. Regulation of hairy-cell survival through constitutive activation of mitogen-activated protein kinase pathways. Oncogene. 2003; 22:2272-84. [PubMed: 12700663]

15. Curtin JA, Fridlyand J, Kageshita T, et al. Distinct sets of genetic alterations in melanoma. N Engl J Med. 2005; 353:2135-47. [PubMed: 16291983]

16. Puxeddu E, Moretti S, Elisei R, et al. BRAF(V599E) mutation is the leading genetic event in adult sporadic papillary thyroid carcinomas. J Clin Endocrinol Metab. 2004; 89:2414-20. [PubMed: 15126572]

17. Badalian-Very G, Vergilio JA, Degar BA, et al. Recurrent BRAF mutations in Langerhans cell histiocytosis. Blood. 2010; 116:1919-23. [PubMed: 20519626]

18. Brose MS, Volpe P, Feldman M, et al. BRAF and RAS mutations in human lung cancer and melanoma. Cancer Res. 2002; 62:6997-7000. [PubMed: 12460918]

19. Tie J, Gibbs P, Lipton L, et al. Optimizing targeted therapeutic development: Analysis of a colorectal cancer patient population with the BRAFV600E mutation. Int J Cancer. $2010 \mathrm{Jul} 15$. [Epub ahead of print].

20. Case M, Matheson E, Minto L, et al. Mutation of genes affecting the RAS pathway is common in childhood acute lymphoblastic leukemia. Cancer Res. 2008; 68:6803-9. [PubMed: 18701506]

21. Gustafsson B, Angelini S, Sander B, et al. Mutations in the BRAF and N-ras genes in childhood acute lymphoblastic leukaemia. Leukemia. 2005; 19:310-2. [PubMed: 15538400]

22. Davidsson J, Lilljebjorn H, Panagopoulos I, et al. BRAF mutations are very rare in B- and T-cell pediatric acute lymphoblastic leukemias. Leukemia. 2008; 22:1619-21. [PubMed: 18273045]

23. Lee JW, Yoo NJ, Soung YH, et al. BRAF mutations in non-Hodgkin's lymphoma. Br J Cancer. 2003; 89:1958-60. [PubMed: 14612909]

24. Keshet Y, Seger R. The MAP kinase signaling cascades: a system of hundreds of components regulates a diverse array of physiological functions. Methods Mol Biol. 2010; 661:3-38. [PubMed: 20811974] 
25. Niault TS, Baccarini M. Targets of Raf in tumorigenesis. Carcinogenesis. 2010; 31:1165-74. [PubMed: 20047953]

26. Wan PT, Garnett MJ, Roe SM, et al. Mechanism of activation of the RAF-ERK signaling pathway by oncogenic mutations of B-RAF. Cell. 2004; 116:855-67. [PubMed: 15035987]

27. Slupsky JR, Kamiguti AS, Harris RJ, et al. Central role of protein kinase Cepsilon in constitutive activation of ERK1/2 and Rac1 in the malignant cells of hairy cell leukemia. Am J Pathol. 2007; 170:745-54. [PubMed: 17255340]

28. Xia Z, Dickens M, Raingeaud J, et al. Opposing effects of ERK and JNK-p38 MAP kinases on apoptosis. Science. 1995; 270:1326-31. [PubMed: 7481820]

29. Bosch F, Campo E, Jares P, et al. Increased expression of the PRAD-1/CCND1 gene in hairy cell leukaemia. Br J Haematol. 1995; 91:1025-30. [PubMed: 8547115]

30. Miranda RN, Briggs RC, Kinney MC, et al. Immunohistochemical detection of cyclin D1 using optimized conditions is highly specific for mantle cell lymphoma and hairy cell leukemia. Mod Pathol. 2000; 13:1308-14. [PubMed: 11144927]

31. Chilosi M, Chiarle R, Lestani M, et al. Low expression of $\mathrm{p} 27$ and low proliferation index do not correlate in hairy cell leukaemia. Br J Haematol. 2000; 111:263-71. [PubMed: 11091210]

32. Roovers K, Davey G, Zhu X, et al. Alpha5beta1 integrin controls cyclin D1 expression by sustaining mitogen-activated protein kinase activity in growth factor-treated cells. Mol Biol Cell. 1999; 10:3197-204. [PubMed: 10512860]

33. Bhatt KV, Spofford LS, Aram G, et al. Adhesion control of cyclin D1 and p27Kip1 levels is deregulated in melanoma cells through BRAF-MEK-ERK signaling. Oncogene. 2005; 24:345971. [PubMed: 15735667]

34. Bhatt KV, Hu R, Spofford LS, Aplin AE. Mutant B-RAF signaling and cyclin D1 regulate Cks1/Sphase kinase-associated protein 2-mediated degradation of p27Kip1 in human melanoma cells. Oncogene. 2007; 26:1056-66. [PubMed: 16924241]

35. Nicolaou F, Teodoridis JM, Park H, et al. CD11c gene expression in hairy cell leukemia is dependent upon activation of the proto-oncogenes ras and junD. Blood. 2003; 101:4033-41. [PubMed: 12576324]

36. Piris, M.; Foucar, K.; Mollejo, M., et al. Splenic B-cell lymphoma/leukaemia, unclassificable. In: Swerdlow, SHea, editor. WHO classification of tumours of haematopoietic and lymphoid tissues. 4. Lyon: International Agency for Research on Cancer (IARC); 2008.

37. Tsai J, Lee JT, Wang W, et al. Discovery of a selective inhibitor of oncogenic B-Raf kinase with potent antimelanoma activity. Proc Natl Acad Sci U S A. 2008; 105:3041-6. [PubMed: 18287029]

38. Sala E, Mologni L, Truffa S, et al. BRAF silencing by short hairpin RNA or chemical blockade by PLX4032 leads to different responses in melanoma and thyroid carcinoma cells. Mol Cancer Res. 2008; 6:751-9. [PubMed: 18458053]

39. Bollag G, Hirth P, Tsai J, et al. Clinical efficacy of a RAF inhibitor needs broad target blockade in BRAF-mutant melanoma. Nature. 2010; 467:596-9. [PubMed: 20823850]

40. Flaherty KR, Keith T, Puzanov I, et al. Inhibition of mutated, activated BRAF in metastatic melanoma. The New England Journal of Medicine. 2010; 363:809-19. [PubMed: 20818844] 


\section{Filtering Pipeline}

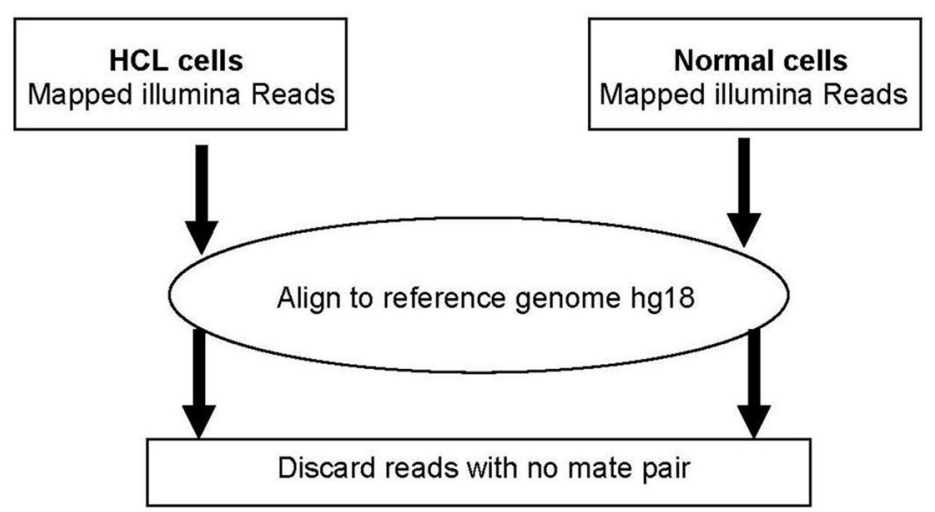

Discard nucleotides with phred $<5$

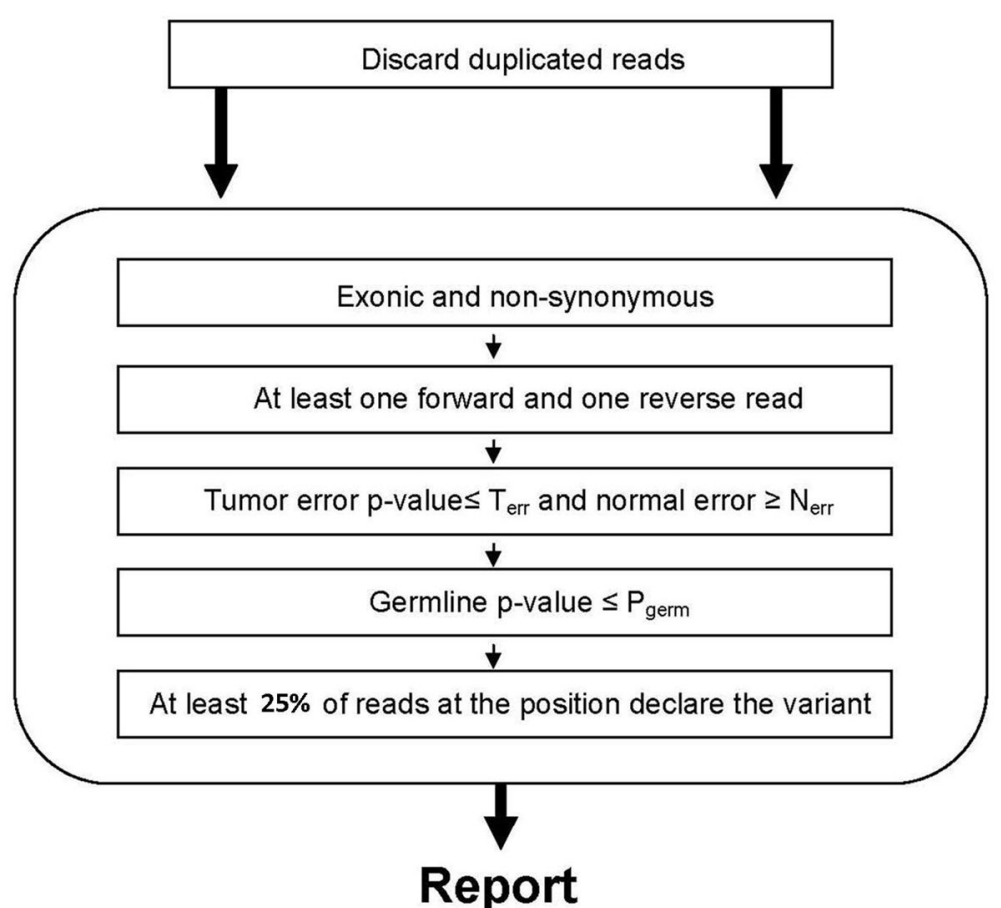

Figure 1. Bioinformatics pipeline for the identification of somatic mutations

After aligning the Illumina reads from the HCL and the normal cell DNA to the human reference genome a series of filters were applied to discard reads not usable for the downstream purpose of somatic mutation discovery. Sequence variants fulfilling the further 5 criteria indicated in the bottom rectangle were subjected to Sanger sequencing validation. See Supplementary Materials for details. 

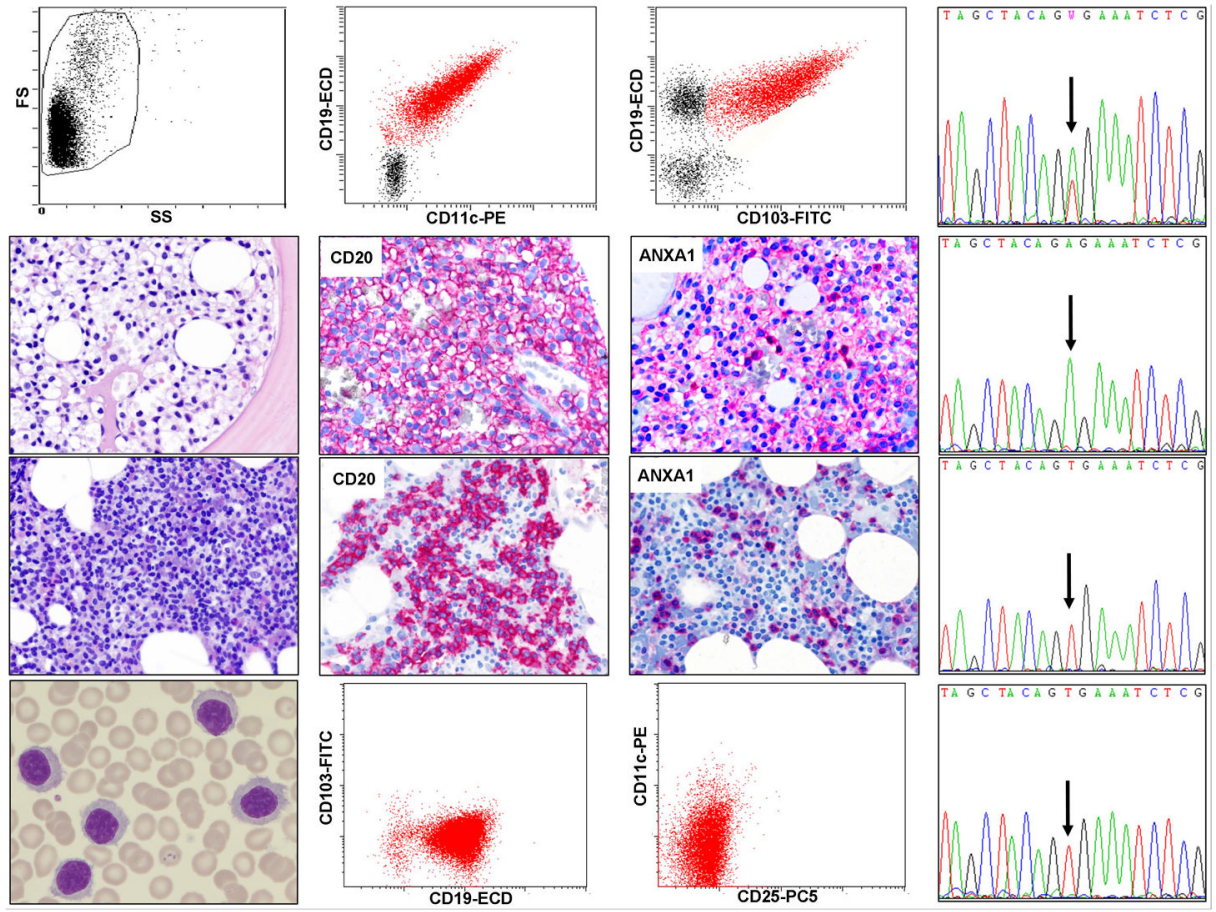

Figure 2. BRAF V600E mutation in HCL but not in other peripheral B-cell neoplasms

Top panels. Flow cytometry analysis of a HCL patient shows peripheral blood mononuclear cells partly with high forward and side scatter features (black events in the dot plot on the far left), expressing CD19 together with CD11c and with CD103 (red events in the middle and right dot plot, respectively). Direct DNA Sanger sequencing of purified leukemic cells reveals a heterozygous T->A mutation (far right; arrow)

Upper middle panels. Another HCL case analyzed in paraffin sections from a bone marrow biopsy (x 400). Diffuse marrow infiltration by HCL cells (far left; hematoxylin \& eosin staining) that display positivity for CD20 (stain in the middle) and for ANXA1 (stain on the right). Purified HCL cells harbor a homozygous/hemizygous T->A mutation (far right; arrow).

Lower middle panels. Splenic Lymphoma/Leukemia Unclassifiable (paraffin sections from a bone marrow biopsy; $x$ 400). Marked marrow infiltration by leukemic cells (far left; hematoxylin \& eosin staining), that express CD20 (stain in the middle) but not ANXA1 (stain on the right, where positive myeloid cells act as internal control). Purified leukemic cells do not carry the T->A mutation (far right; arrow).

Bottom panels. Splenic marginal zone lymphoma. Leukemic cells in the peripheral blood smear show the typical morphological features with polar villi (far left; May-GrünwaldGiemsa staining, $x$ 630). At flow cytometry, leukemic cells express CD19 but not CD103 (red events in the left dot plot). These cells only weakly express CD11c and are negative for CD25 (red events in the right dot plot). Leukemic cells do not carry the T->A mutation (far right; arrow). 

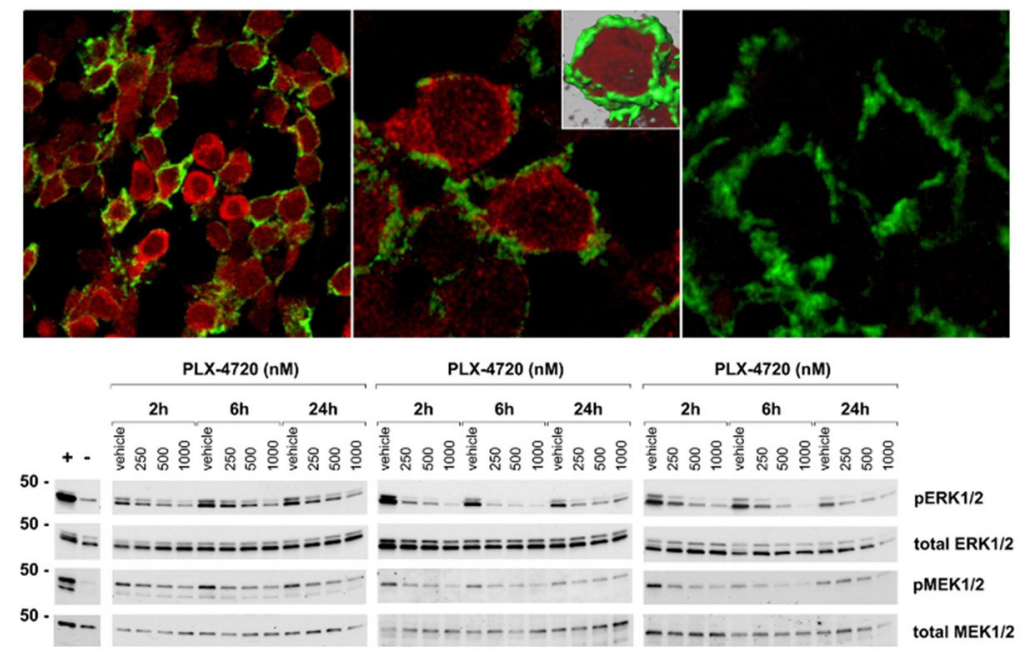

Figure 3. Expression of phospho-MEK and phospho-ERK by HCL cells

Top panels: Double immunofluorescence staining for CD20 (green) and phospho-ERK (red) in paraffin sections from a bone marrow trephine. The panels show the expression of phospho-ERK in CD20-positive leukemic hairy cells at lower (left) and higher magnification (middle). The inset shows a 3D reconstruction of confocal slices of a representative cell with the "isosurface" tecnique. The right panel shows that staining for phospho-ERK is completely blocked by pre-incubation of the antibody with the specific phospho-ERK peptide.

Bottom panels: Western blot analysis on purified HCL cells from 3 representative patients showing phosphorylation of both MEK and ERK kinases under basal conditions (vehicle treatment) and their dose-dependent dephosphorylation after 2, 6 and $24 \mathrm{~h}$ incubation with the specific active BRAF inhibitor PLX-4720 at $250 \mathrm{nM}, 500 \mathrm{nM}$ or $1000 \mathrm{nM}$ concentrations. Membranes were probed with antibodies against phospho-ERK, phosphoMEK, total ERK and total MEK, as indicated on the far right. Protein lysates from either serum-starved or unstarved human leukemic Jurkat T cell line cells were used as negative (-) and positive (+) control, respectively, for ERK and MEK phosphorylation. Numbers on the left indicate molecular weight markers (in $\mathrm{kDa}$ ). 


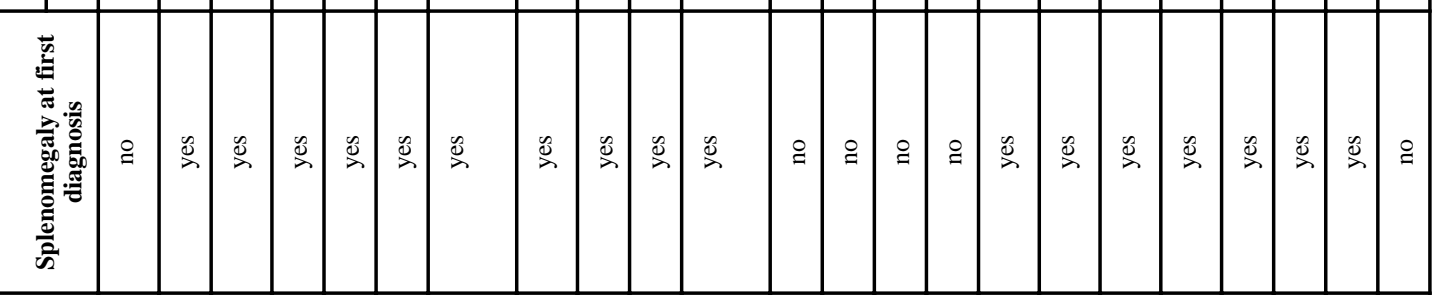

출

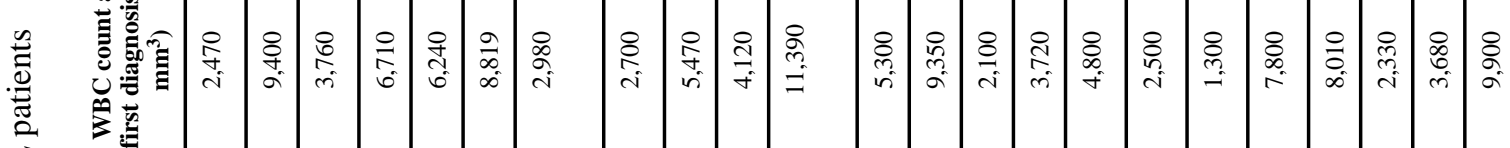

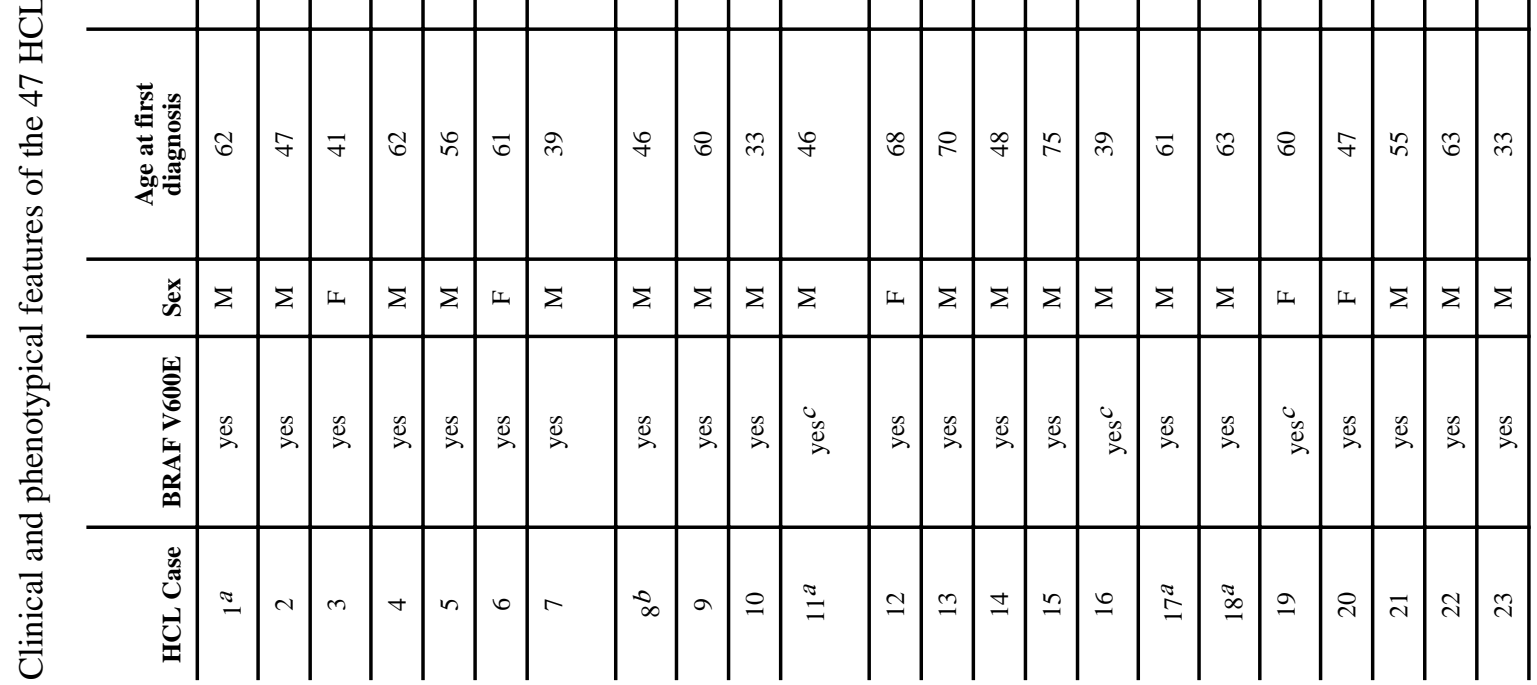




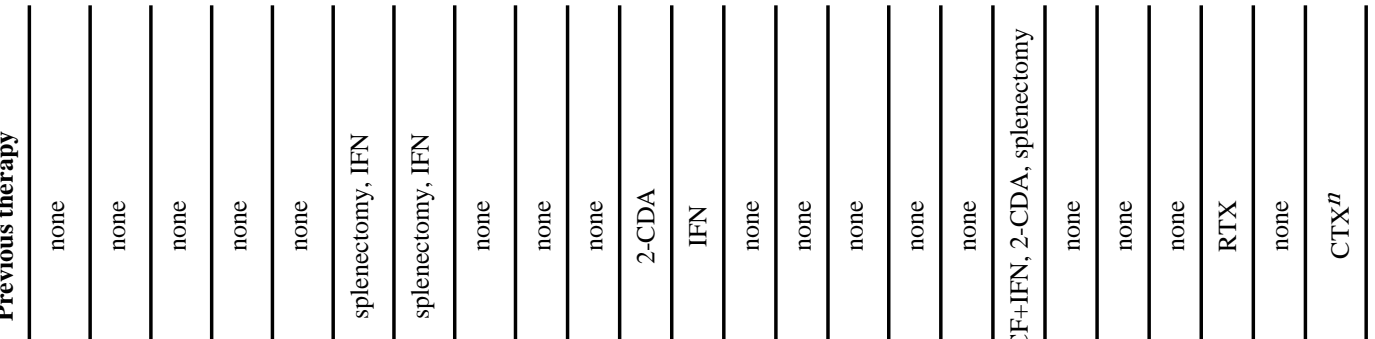

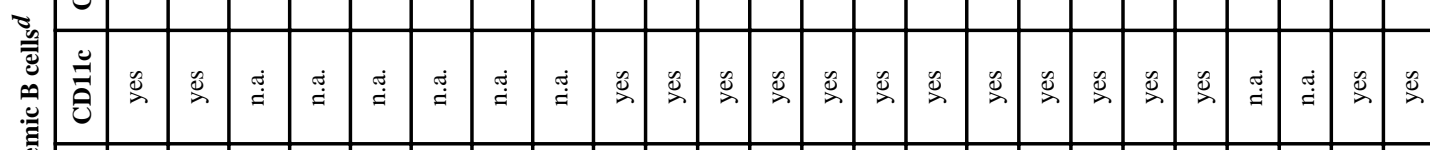

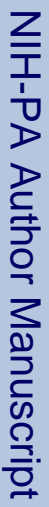

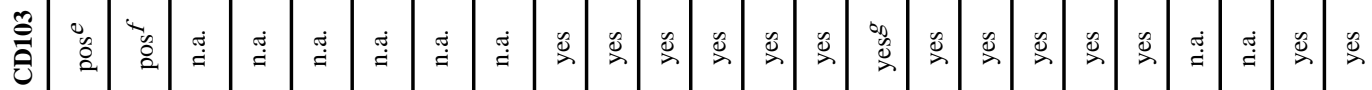

产

赵

它

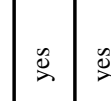

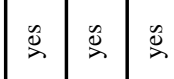

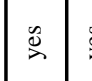

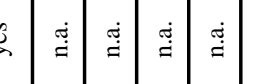

$\stackrel{\oplus}{=} \stackrel{\oplus}{=}$

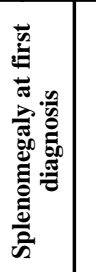

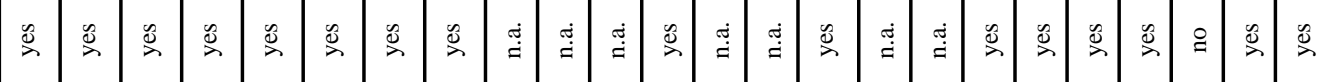

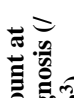

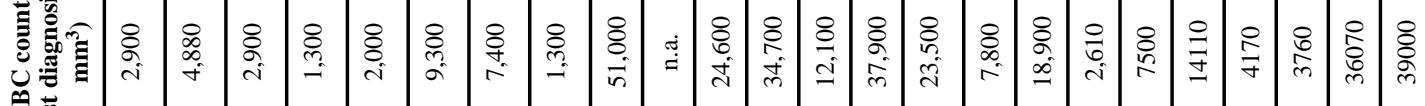

要

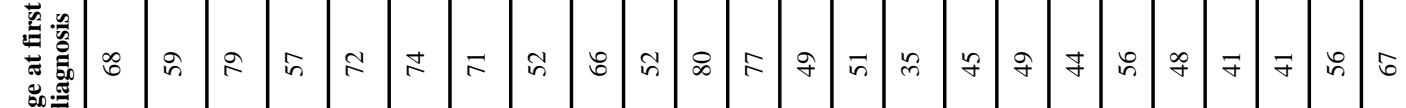

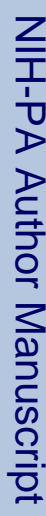

(5)

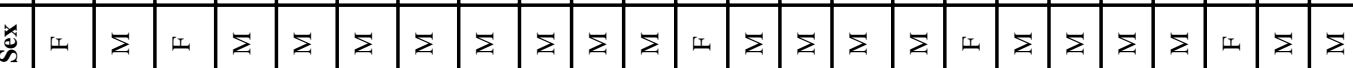

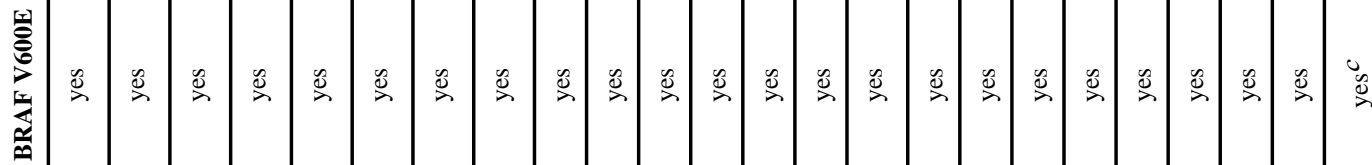

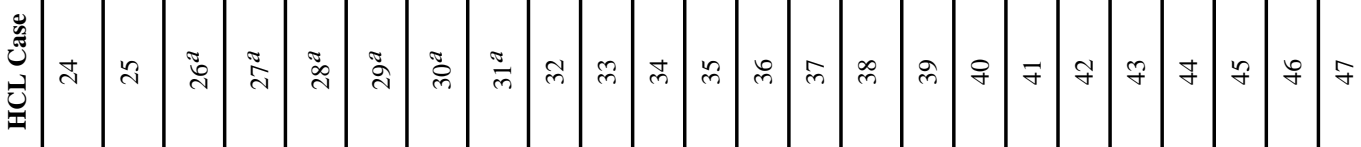

$\ddot{\hat{\sigma}}$
$\stackrel{\leftrightarrow}{\mathrm{g}}$

高 


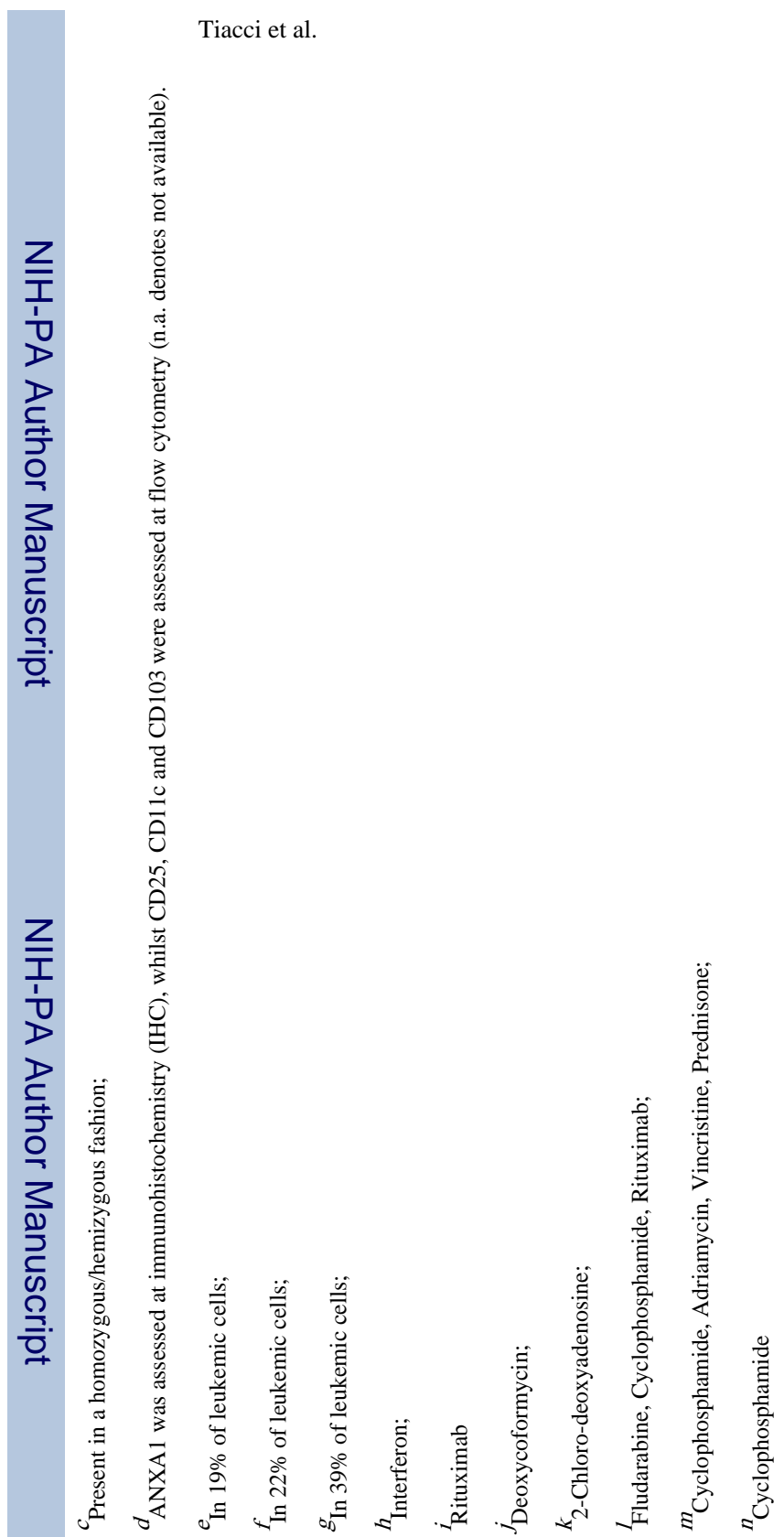

N Engl J Med. Author manuscript; available in PMC 2013 June 21. 